\title{
Testing geovisualisations for effective environmental engineering decision-making
}

\author{
Jess Hepburn ${ }^{a,} *$, David Fairbairn ${ }^{b}$ \\ ${ }^{a}$ Newcastle University, Newcastle upon Tyne,UK; j.k.hepburn1@newcastle.ac.uk \\ ${ }^{b}$ Newcastle University, Newcastle upon Tyne,UK; david.fairbairn@newcastle.ac.uk \\ * Corresponding author
}

Keywords: Environmental Impact Assessment (EIA), spatial decision making, geovisualisation

Within large engineering consultancies, multi-disciplinary projects are common, incorporating large volumes of varied and volatile high-dimensional data, usually geospatial in nature, and often uncertain. Environmental Impact Assessments (EIAs), legally embedded in engineering practice as an EU directive, use a combination of data types, sourced from numerous disciplines. These provide information for stakeholders, including planners, local authorities, and the public, in projects which affect neighbourhoods, districts, and landscapes. Contemporary geovisualisations can incorporate and help use of the outputs from a variety of analysis and models, enhancing decision-making processes inherent in all engineering projects.

Treating geovisualisations as a formal communication tool allows for improved map and graphical understanding, reducing the loss of information within the communication system (MacEachren, 1995). Therefore, it is important to consider geovisualisation as a critical element of the whole information retrieval and decision-making process. Decisions within the EIA process tend to assess the likely significant environmental effects arising from proposed development, in a systematic, data informed system, allowing for a reduction in risk of damage to the environment (Town and Country Planning Regulations, 2011).

Much of the data generated for EIA is processed through models and analysis, meaning there are margins of error and uncertainty present in the data. Traditional cartographic methods can be effectively utilised in the representation of such uncertainty (MacEachren, 1992). Incorporating uncertainty in a geovisualisation allows the viewer to interpret information about the data, rather than just observe location and attributes.

This study considers four main themes: cognition (decision making), visualisation, risk and ElA. Although discrete, these all contribute to decision making in an engineering consultancy (Figure 1). A major aim is to understand how users interact with spatial data, in terms of considering the role of uncertainty in decision making within industrial practice, and assess methods of visualising such uncertainty. This requires investigation of user interaction with such visual, graphical and map-based representations.

Eye tracking using the Tobii $\times 2-30$ will be the main method used to evaluate user interaction. Such experimental testing reflects vision as the strongest of the senses in humans, revealing both physiological eye movements and also reflecting a subconscious representation of the user's internal thoughts (Çöltekin et al., 2010). Unlike some user interaction testing methods, eye movement recordings do not rely on selfreporting, and can thus be considered objective, as well as being used to enhance traditional performance tests (Goldberg and Kotval, 1999). Eye tracking, plus the recording of keyboard and mouse clicks and recording of screen images, will be monitored to understand the user's subliminal interaction with the data.

For this focussed and discipline-specific interaction with spatial data, user knowledge will be assessed and recorded: this important factor has been recognised through many study results (e.g. Kobus et al., 2001; Hope and Hunter, 2007) which suggest that the knowledge of users has a major impact on their decision- 
making abilities and outcomes. The user knowledge and experience, recorded in an individual personal profile and examined in conjunction with the environmental and engineering decisions made, will be collected through an Esri Survey123 online document prior to the main interaction testing.

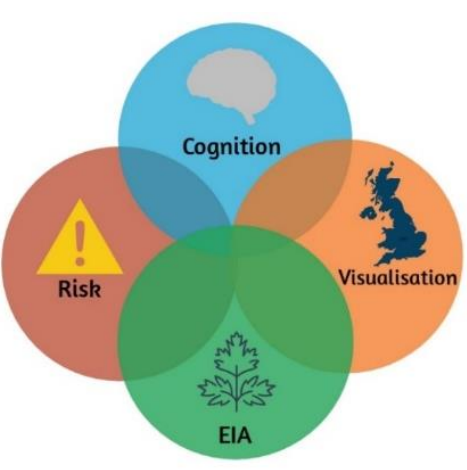

Fig 1: 4 main themes in this study

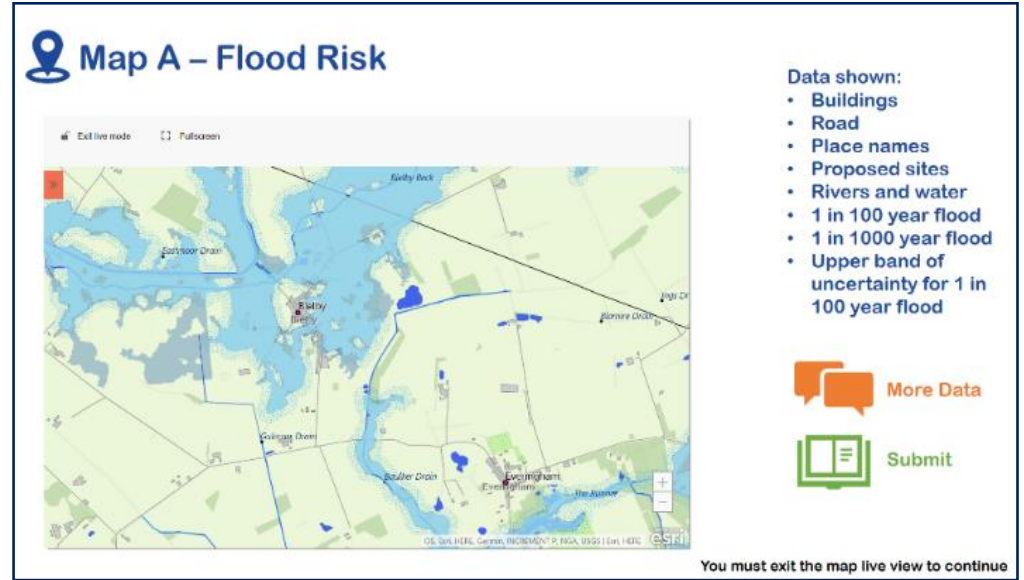

Fig 2: sample interface to engineering project mitigating rural flood risk

Geovisualisations will be displayed in an online interface, accessible in the users' 'typical' working environment, through their standard desktop device. Avoiding novel and unique interactions (e.g. virtual reality headsets, immersive decision theatres) which may reveal interesting but irreproducible results, the regular day-to-day environment for those engineers who are charged with planning and implementing major infrastructure and environmental projects is used, giving a realistic impression of their working practices, and triggering decisions to be made which are similar to those usually made by the user. There will be no artificial environmental control during the study, with users able to pan, zoom and navigate the interface, switch on and off available layers, undertake analysis to create and incorporate added-value, interrogate data through the map interface, add their own data and develop scenarios, and in all senses interact freely with the geovisualisation (exemplified in Figure 2). Importantly, opportunities to assess data uncertainty, both implicitly and explicitly, will be incorporated. Each test should take no more than 20 minutes, with users being asked to make decisions based on the data available, the task required, and their own interpretation of the scenario.

Three case studies will be presented to the user, covering typical arenas where decision making and EIA within an engineering consultancy are interacting: a rural study relates to flood risk, an urban example addresses traffic risk near a school, and an environmentally sensitive area must consider environmental risk from the construction of a new cable route.

Once data is collected from between 30 and 40 participants, it will be analysed through established areas of interest (eAOI), used to categorise the points of gaze, fixation time, fixation count and saccades through Tobii Pro Studio. Capture of associated screen and keyboard interactions will also be incorporated, with statistical tests being used to establish the significance in the results.

It is hoped that the results from this study will help assist engineering consultancies, allowing for collaboration between teams, and streamlining of decision making, resulting in better practice. By discussing and considering the four discrete themes of cognition, risk, visualisation and environmental impact assessments, the research is intended to develop a rounded and informed approach for identifying issues and establishing best practice for common industry tasks. 